\title{
A SIMPLE PROOF OF GOPAKUMAR-VAFA CONJECTURE FOR LOCAL TORIC CALABI-YAU MANIFOLDS
}

\author{
PAN PENG \\ Department of Mathematics, UCLA
}

\begin{abstract}
We prove Gopakumar-Vafa conjecture for local toric CalabiYau manifolds. It is also proved that the local Goparkumar-Vafa invariants of a given class at large genus vanish.
\end{abstract}

\section{INTRODUCTION}

Let $X$ be a Calabi-Yau threefold. $K_{\Sigma}^{g}(X)$ is defined as the genus $g$, 0 marked point Gromov-Witten invariant of $X$ in the curve class $\Sigma \in$ $H_{2}(X, \mathbb{Z})$. From M-theory, Gopakumar and Vafa [7] express the invariants $K_{\Sigma}^{g}(X)$ in terms of integer invariants $N_{\Sigma}^{g}(X)$ obtained by BPS state counts . In the physics literature, these invariants should be the Euler characteristics of moduli space of D-brane. Unfortunately, this moduli space has not been defined so far in mathematics. We will take the following Gopakumar-Vafa formula as the definition of Gopakumar-Vafa invariant $N_{\Sigma}^{g}(X)$.

(1) $\sum_{\Sigma \neq 0} \sum_{g \geq 0} K_{\Sigma}^{g}(X) t^{2 g-2} e^{-\omega \cdot \Sigma}=\sum_{\Sigma \neq 0} \sum_{n \geq 1} \sum_{g \geq 0} \frac{N_{\Sigma}^{g}(X)}{n}\left(2 \sin \left(\frac{n t}{2}\right)\right)^{2 g-2} e^{-n \omega \cdot \Sigma}$

One can recursively obtain Gopakumar-Vafa invariants from Gromov-Witten invariants through the above formula and vice versa. It's not hard to see that Gopakumar-Vafa invariants are rational number from the expression. The following Gopakumar-Vafa conjecture captures our intensive interests.

Conjecture 1.1. (Gopakumar-Vafa) $\forall \Sigma \in H_{2}(X, \mathbb{Z})$ and $g \geq 0, N_{\Sigma}^{g} \in$ $\mathbb{Z}$.

From (11), there is no reason (at least mathematically) that these $N_{\Sigma}^{g}$ 's should be integers.

In general, in order to obtain Gopakumar-Vafa invariants from GromovWitten invariants, one has to get a way to compute the corresponding topological string amplitudes. For lower genus, one may use mirror symmetry or localization method. However, if one goes to higher genus, the calculation become more and more complicated. Neither of these two methods is very practical. This situation has changed since a beautiful discovery

Date: 10/18/2004. 
of the duality between Chern-Simons theory and Gromov-Witten theory [8]. Chern-Simons theory provides a very efficient way to compute all genus amplitudes for toric Calabi-Yau manifolds.

Recently, in 12 J. Li, C.-C. Liu, K. Liu and J. Zhou build the mathematical foundation of topological vertex theory, which allows us to rigorously write down all genus amplitudes for any toric Calabi-Yau manifold directly from its toric diagram. Thus it may provide us a new way to approach Gopakumar-Vafa conjecture. For physics literature about topological vertex, one may refer [1].

In this paper, we consider the local toric Calabi-Yau geometry, which is the geometry of Fano surfaces within Calabi-Yau threefold. The corresponding all genus A-model amplitudes obtained from its relation to Chern-Simons amplitudes can be organized in a simple way. In local Calabi-Yau, a tubular neighborhood of the Fano surface is equivalent to the total space of the canonical bundle. Note that local Calabi-Yau manifolds are noncompact.

Let $S$ be a nonsingular, projective, toric, Fano surface and denote by $K_{S}$ its canonical bundle. The Gromov-Witten theory of local Calabi-Yau geometry of $S$ is defined by excess integral. Let $\bar{M}_{g}(S, \Sigma)$ denote the moduli space of stable maps from connected genus g curves with 0 marked point to $S$ representing the class $\Sigma \in H_{2}(S, \mathbb{Z})$. The local $(\Sigma, g)$ Gromov-Witten invariant of $S$ is defined by

$$
K_{\Sigma}^{g}(S)=\int_{\left[\bar{M}_{g}(S, \Sigma)\right]^{v i r}} e\left(R^{1} \pi_{*} e v^{*} K_{S}\right)
$$

Where $\pi: \mathcal{U} \rightarrow \bar{M}_{g}(S, \Sigma)$ is the universal curve, ev $: \bar{M}_{g}(S, \Sigma) \rightarrow S$ is the evaluation map. We still take the formula (11) as the definition of local Gopakumar-Vafa invariant $N_{\Sigma}^{g}(S)$ while we use the corresponding local Gromov-Witten invariant $K_{\Sigma}^{g}(S)$ instead.

However, the invariant $\widehat{N}_{\Sigma}^{g}(S)$ we mention later as local Gopakumar-Vafa invariant defers slightly from the above. The relation between $\widehat{N}_{\Sigma}^{g}(S)$ and $N_{\Sigma}^{g}(S)$ is $\widehat{N}_{\Sigma}^{g}(S)=(-1)^{g-1} N_{\Sigma}^{g}(S)$. We call them both local GopakumarVafa invariants.

In this paper, we proved that for any local toric Calabi-Yau manifold, the Gopakumar-Vafa conjecture is true. More precisely, given any $\Sigma$ as above, we have:

Theorem 5.2 $P_{\Sigma}^{(S)}(x)=\sum_{g \geq 0} \widehat{N}_{\Sigma}^{g}(S) x^{g} \in \mathbb{Z}[x]$.

Before beginning the proof we may note that, from our result, GopakumarVafa invariants vanish at large genera. This is an very interesting phenomenon which is pointed out in [7. After our calculations on Hirzebruch surfaces and $\mathbb{P}^{2}$, we find that the degree of $P_{\Sigma}$ is actually the arithmetic genus $A_{\Sigma}$ of the curve with the degree $\Sigma$. Moreover, $\widehat{N}_{\Sigma}^{A_{\Sigma}}=\chi(L(\Sigma))$, where $L(\Sigma)$ is the holomorphic line bundle associated to the devisor $\Sigma$. All these seems to imply that Gopakumar-Vafa invariants should be related to the embedding curves. 
Our proof of Theorem 5.2 contains two parts. First, we extract GopakumarVafa invariants by proving a generalized Möbius inversion formula (Theorem 4.1). Thus we obtain an explicit formula for $P_{\Sigma}(x)$, while r.h.s of the formula merely contains the Chern-Simons invariants of Hopf links. The standard calculation of Schur function then leads to the following property: $P_{\Sigma}(x)=\frac{1}{c} h(x)$, where $c \in \mathbb{N}, h(x) \in \mathcal{L}[x](\mathcal{L}[x]$ is defined by (12)). Here comes a crucial observation: $P_{\Sigma}(x)$ is a polynomial. In the second part, we show a pattern theorem (Theorem [5.11). Theorem [5.1 asserts if $P_{\Sigma}(x)$ satisfies some conditions which exactly fits our situation, then $P_{\Sigma}(x) \in \mathbb{Z}[x]$. We prove Theorem 5.1 by deliberately pairing the data when multiple covering happens. This pairing shows $c=1$, which is equivalent to $P_{\Sigma} \in \mathcal{L}[x]$. $P_{\Sigma}(x) \in \mathbb{Z}[x]$ then follows from the structure of $\mathcal{L}[x]$.

In our proof, the pattern theorem (Theorem 5.1) doesn't depend on the concrete expression of all genus amplitudes of local toric Calabi-Yau manifolds. The technique we develop here may extend to general toric Calabi-Yau using the topological vertex theory. Now this work is in its preparation.

It is worth mentioning that a new idea of understanding GopakumarVafa conjecture [13] comes out recently. They regard topological string amplitudes as equivariant indices and translate Gopakumar-Vafa conjecture into a formula of an infinite product, where the integrality naturally follows.

The rest of this paper is organized as follows. We fix some notations on partition in section 2. Then we prove some results on symmetric functions and Chern-Simons invariants of the Hopf link in section 3. In section 4, we give an explicit formula for Gopakumar-Vafa invariants. Our main result is proved in section 5. Some examples are discussed in section 6 .

Acknowledgements. I would like to thank my advisor, Professor Kefeng Liu, for his constant encouragement and a lot of inspiring discussions. I am very grateful to Xiaowei Wang for his many helpful comments and friendship. I also would like to thank Professor Jian Zhou for pointing out some misleading parts and some mistakes. At last, I want to thank Professor Amer Iqbal for his explaining me the physical reason of the vanishing property of Gopakumar-Vafa invariants at large genera.

\section{PReliminaries}

2.1. Partitions. A partition is a sequence of non-negative integers

$$
\lambda=\left(\lambda_{1}, \lambda_{2}, \cdots, \lambda_{r}, \cdots\right)
$$

such that

$$
\lambda_{1} \geq \lambda_{2} \geq \cdots \geq \lambda_{r} \geq \cdots
$$

and containing only finitely many non-zero terms. The degree of $\lambda$ is defined by

$$
|\lambda|=\lambda_{1}+\lambda_{2}+\cdots
$$


If $|\lambda|=d$, we denote it by $\lambda \vdash d$. The length of $\lambda$ is defined by $l(\lambda):=$ $\operatorname{Card}\left\{j: \lambda_{j} \neq 0\right\}$. The number $m_{i}(\lambda)=\operatorname{Card}\left\{j: \lambda_{j}=i\right\}$ is called the multiplicity of $i$ in $\lambda$. We may rewrite a partition $\lambda$ as $\left(1^{m_{1}(\lambda)} 2^{m_{2}(\lambda)} \cdots n^{m_{n}(\lambda)} \cdots\right)$. One can identify a partition with its Young diagram or $\left\{(i, j) \in \mathbb{Z}_{+}^{2}: j \leq \lambda_{i}\right\}$. We will freely refer them when needed as long as without any confusion. By transposing the Young diagram of $\lambda$, one gets the corresponding partition $\lambda^{t} . \forall x=(i, j) \in \lambda$, let

$$
c(x)=j-i, \quad h(x)=\lambda_{i}+\lambda_{j}^{t}-i-j+1, \quad n(\lambda)=\sum_{i}(i-1) \lambda_{i} .
$$

For a partition $\lambda$, it's easy to see that $|A u t \lambda|=\prod_{i \geq 1} m_{i}(\lambda)$ !. Define the following constants associated to the partition $\lambda$ :

$$
\begin{aligned}
& u_{\lambda}=\frac{l(\lambda) !}{\prod_{i} m_{i}(\lambda) !}=\frac{l(\lambda) !}{|A u t \lambda|} \\
& z_{\lambda}=\mid \text { Aut } \mid \cdot \prod_{i \geq 1} \lambda_{i} \\
& k_{\lambda}=\sum_{i} \lambda_{i}\left(\lambda_{i}-2 i+1\right) \\
& \theta_{\lambda}=\frac{(-1)^{l(\lambda)-1}(l(\lambda)-1) !}{\mid \text { Aut } \mid}
\end{aligned}
$$

and let $u_{(0)}=\theta_{(0)}=z_{(0)}=1$. Note that $k_{\lambda}=-k_{\lambda^{t}}$ and $k_{\lambda}$ is even.

Lemma 2.1. $\sum_{x \in \lambda} h(x)-\frac{k_{\lambda}}{2}=2 n(\lambda)+|\lambda|$.

Proof.

$$
\begin{aligned}
k_{\lambda} & =\sum_{i} \lambda_{i}\left(\lambda_{i}-2 i+1\right) \\
& =2 \sum_{i} \frac{\lambda_{i}\left(\lambda_{i}-1\right)}{2}-2 \sum_{i} \lambda_{i}(i-1) \\
& =2\left(n\left(\lambda^{t}\right)-n(\lambda)\right)
\end{aligned}
$$

Follow ([16] page 11), one has

$$
\sum_{x \in \lambda} h(x)=n(\lambda)+n\left(\lambda^{t}\right)+|\lambda|
$$

Then direct calculation leads to the proof.

2.2. Infinite series and partition. Given a sequence $x=\left(x_{1}, x_{2}, \cdots, x_{n}, \cdots\right)$, let $|x|=\sum_{i \geq 1} x_{i}$. For a partition $\lambda$, define $x_{\lambda}=\prod_{i \geq 1} x_{\lambda_{i}}, x_{(0)} \equiv 1$.

Lemma 2.2. Let $f(t)=\sum_{n \geq 0} a_{n} t^{n}, x$ is as above. Then

$$
f(|x|)=\sum_{|\lambda| \geq 0} a_{l(\lambda)} x_{\lambda} u_{\lambda}
$$


Proof. Note that $|x|^{d}=\sum_{l(\lambda)=d} x_{\lambda} u_{\lambda}$. The proof then follows from a simple calculation.

Apply the above lemma to $\exp (x)$ and $\log (x)$, we have

\section{Corollary 2.1.}

$$
\begin{aligned}
\exp \left(\sum_{n \geq 1} b_{n} x^{n}\right) & =1+\sum_{|\lambda| \geq 1} \frac{b_{\lambda} x^{|\lambda|}}{|A u t \lambda|} \\
\log \left(1+\sum_{n \geq 1} b_{n} x^{n}\right) & =\sum_{|\lambda| \geq 1} b_{\lambda} x^{\lambda} \theta_{\lambda}
\end{aligned}
$$

\section{Symmetric Functions}

3.1. Basis. Let $x=\left(x_{1}, x_{2}, \cdots\right)$ be a sequence, $\lambda$ a partition. Denote by $\operatorname{Per}(\lambda)=\{\delta: \delta$ is a permutation of $\lambda\}$. Follow the traditional notations:

$$
m_{\lambda}=\sum_{\delta \in \operatorname{Per}(\lambda)} x^{\delta}, \quad p_{n}=m_{(n)}, \quad h_{n}=\sum_{\lambda \vdash n} m_{\lambda}
$$

Let $p_{\lambda}=\prod_{i} p_{\lambda_{i}}$. Similarly define $h_{\lambda}$. Schur function $S_{\lambda}=\operatorname{det}\left(h_{\lambda_{i}-i+j}\right)_{1 \leq i, j \leq l(\lambda)}$. It's easy to see that

$$
H(x, t)=\sum_{r \geq 0} h_{r} t^{r}=\prod_{i}\left(1-x_{i} t\right)^{-1}
$$

3.2. Chern-Simons invariants of the Hopf link $\mathcal{W}_{\lambda \mu}(q)$. Define:

$$
\mathcal{W}_{\lambda}(q)=q^{\frac{k_{\lambda}}{4}} \prod_{1 \leq i<j \leq d} \frac{\left[\lambda_{i}-\lambda_{j}+j-i\right]}{[j-i]} \prod_{i=1}^{l(\lambda)} \prod_{v=1}^{\lambda_{i}} \frac{1}{[v-i+l(\lambda)]}
$$

and

$$
\mathcal{W}_{\lambda \mu}(q)=\mathcal{W}_{\lambda}(q) S_{\mu}\left(q^{\lambda_{1}-\frac{1}{2}}, q^{\lambda_{2}-\frac{3}{2}}, \cdots, q^{\lambda_{n}-n+\frac{1}{2}}, \cdots\right) .
$$

$\mathcal{W}_{\lambda}(q)$ can be simplified as

$$
\mathcal{W}_{\lambda}(q)=q^{\frac{k_{\lambda}}{4}} \prod_{x \in \lambda} \frac{1}{[h(x)]}=S_{\lambda}\left(q^{-\frac{1}{2}}, q^{-\frac{3}{2}}, \cdots, q^{-n+\frac{1}{2}}, \cdots\right)
$$

where $[m]=q^{\frac{m}{2}}-q^{-\frac{m}{2}}$. Hence

$$
\mathcal{W}_{\lambda \mu}(q)=q^{-\frac{\lambda+\mu}{2}} S_{\lambda}\left(q^{\alpha}\right) S_{\mu}\left(q^{\lambda+\alpha}\right)
$$

where $q^{\alpha}=\left(1, q^{-1}, q^{-2}, \cdots\right)$. It is proved in [19] that

$$
\mathcal{W}_{\lambda^{t} \mu^{t}}(q)=(-1)^{|\lambda|+|\mu|} \mathcal{W}_{\lambda \mu}\left(\frac{1}{q}\right)
$$


Follow (16 page 27)

$$
\begin{aligned}
H\left(q^{\alpha}, t\right) & =\prod_{i=1}^{\infty}\left(1-q^{-i+1} t\right)^{-1} \\
& =\sum_{r=0}^{\infty} \frac{t^{r}}{\phi_{r}\left(\frac{1}{q}\right)} \\
& =\sum_{r=0}^{\infty} \frac{\phi_{r}\left(\frac{1}{q}\right) q^{\frac{r(r+1)}{2}}}{\prod_{i=1}^{r}[i]^{2}} t^{r}
\end{aligned}
$$

where $\phi_{r}(q)=\prod_{i=1}^{r}\left(1-q^{i}\right)$. Then correspondingly

$$
h_{r}\left(q^{\alpha}\right)=\frac{\phi_{r}\left(\frac{1}{q}\right) q^{\frac{r(r+1)}{2}}}{\prod_{i=1}^{r}[i]^{2}}
$$

Using the results of $H\left(q^{\alpha}, t\right)$, we can calculate $H\left(q^{\lambda+\alpha}, t\right)$ in the following way:

$$
\begin{aligned}
H\left(q^{\lambda+\alpha}, t\right) & =\prod_{i=1}^{\infty}\left(1-q^{\lambda_{i}-i+1} t\right)^{-1} \\
& =\prod_{i=1}^{l(\lambda)} \frac{1-q^{-i+1} t}{1-q^{\lambda_{i}-i+1} t} H\left(q^{\alpha}, t\right) \\
& =\prod_{i=1}^{l(\lambda)} \frac{1-q^{-i+1} t}{1-q^{\lambda_{i}-i+1} t} \sum_{r=0}^{\infty} \frac{\phi_{r}\left(\frac{1}{q}\right) q^{\frac{r(r+1)}{2}}}{\prod_{i=1}^{r}[i]^{2}}
\end{aligned}
$$

Observe that one can expand $\left(1-q^{\lambda_{i}-i+1} t\right)^{-1}$ and get the coefficient of $t^{j}$, which is an element of $\mathbb{Z}\left[q, q^{-1}\right]$. After collecting the $t^{r}$ terms in the above formula, we have

$$
h_{r}\left(q^{\lambda+\alpha}\right)=\frac{\rho(q)}{\prod_{i=1}^{r}[i]^{2}}
$$

Here $\rho(q) \in \mathbb{Z}\left[q, q^{-1}\right]$. Recall $S_{\lambda}=\operatorname{det}\left(h_{\lambda_{i}-i+j}\right)_{1 \leq i, j \leq l(\lambda)}$. From Lemma 3.3. we know $[n]^{2}$ is a polynomial of $[1]^{2}$ with integer coefficients. Combine (6) and (77), we obtain:

Lemma 3.1. $S_{\lambda}\left(q^{\alpha}\right), S_{\mu}\left(q^{\lambda+\alpha}\right)$ are of form $\frac{a(q)}{b\left([1]^{2}\right)}$, where $a(q) \in \mathbb{Z}\left[q, q^{-1}\right]$, $b(x) \in \mathbb{Z}[x]$ and is monic.

Let $\mathcal{P}$ denote the space of all partitions. Define

$$
\mathcal{W}_{\vec{R}, \gamma}=\mathcal{W}_{R^{N} R^{1}} \mathcal{W}_{R^{1} R^{2}} \mathcal{W}_{R^{2} R^{3}} \cdots \mathcal{W}_{R^{N-1} R^{N}}(-1)^{\sum_{i=1}^{N} \gamma_{i}\left|R^{i}\right|} q^{\frac{1}{2} \sum_{i=1}^{N} k_{R^{i}} \gamma_{i}}
$$

where $\vec{R}=\left(R^{1}, R^{2}, \cdots, R^{N}\right) \in \mathcal{P}^{N}$ and $\gamma=\left(\gamma_{1}, \gamma_{2}, \cdots, \gamma_{N}\right) \in \mathbb{Z}^{N}$. Denote by $\|\vec{R}\|=\sum_{i=1}^{N}\left|R^{i}\right|$ and $\overrightarrow{R^{t}}=\left(\left(R^{1}\right)^{t},\left(R^{2}\right)^{t}, \cdots,\left(R^{N}\right)^{t}\right)$. Let $R^{N+1}=R^{1}$, 
we rewrite $\mathcal{W}_{\vec{R}, \gamma}$ as

$$
\begin{aligned}
\mathcal{W}_{\vec{R}, \gamma}= & q^{-\sum_{i=1}^{N}\left|R^{i}\right|} \prod_{i=1}^{N} S_{R^{i}}\left(q^{\alpha}\right) \prod_{i=2}^{N+1} S_{R^{i}}\left(q^{R^{i-1}+\alpha}\right) \\
& \times(-1)^{\sum_{i=1}^{N} \gamma_{i}\left|R^{i}\right|} q^{\frac{1}{2} \sum_{i=1}^{N} k_{R^{i}} \gamma_{i}}
\end{aligned}
$$

Notice that $k_{\lambda}$ is even for any partition $\lambda$, from (8) and lemma 3.1 we have

Corollary 3.1. $\mathcal{W}_{\vec{R}, \gamma}$ is of form $\frac{a(q)}{b\left([1]^{2}\right)}$, where $a(q) \in \mathbb{Z}\left[q, q^{-1}\right], b(x) \in \mathbb{Z}[x]$ and is monic.

Lemma 3.2. $\mathcal{W}_{\vec{R}^{t}, \gamma}(q)=\mathcal{W}_{\vec{R}, \gamma}\left(\frac{1}{q}\right)$.

Proof. Before proving the lemma, let's look at the following statement first.

Lemma 3.3. $f(q) \in \mathbb{Z}\left[q, q^{-1}\right], f(q)=f\left(q^{-1}\right)$. Then $f \in \mathbb{Z}[x]$, where $x=[1]^{2}$.

Proof. $f(q)=f\left(q^{-1}\right)$, and $f \in \mathbb{Z}\left[q, q^{-1}\right] . f$ has to be of the form $\sum_{n=0}^{N} a_{n}\left(q^{n}+\right.$ $q^{-n}$ ) for some $N$. It's sufficient to show $\forall n, q^{n}+q^{-n} \in \mathbb{Z}[x]$.

$n=1, q^{1}+q^{-1}=x+2$. $n=k$. If $k$ odd, $q^{n}+q^{-n}=\left(q+q^{-1}\right)\left(q^{k-1}+\right.$ $\left.q^{k-2}+\cdots+q^{-(n-1)}\right)$; if $k$ even, $q^{n}+q^{-n}=\left(q^{\frac{n}{2}}+q^{\frac{-n}{2}}\right)^{2}-2$. So by induction, the proof is completed.

From lemma 3.3 and corollary 3.1 the proof of lemma 3.2 follows easily from the formula (5).

\section{Local Gopakumar-Vafa invariants}

4.1. A generalized Möbius inversion formula. Let $\mu(n)$ be the Möbius function. That is:

$$
\mu(n)= \begin{cases}(-1)^{r}, & n=p_{1} \cdot p_{2} \cdots p_{r} \text { and } p_{i} \neq p_{j}, \quad \text { if } i \neq j \\ 0, & \text { otherwise. }\end{cases}
$$

Lemma 4.1. $\sum_{k \mid n} \mu(k)=\delta_{1 n}$

Proof. $n=1$, trivial. If $n>1$, say $n=\prod_{i=1}^{d} p_{i}^{r_{i}}, p_{i}$ 's are distinct and $r_{i}>0$. Then we have

$$
\sum_{k \mid d} \mu(k)=\sum_{i=0}^{d}\left(\begin{array}{l}
d \\
i
\end{array}\right)(-1)^{i}=(1-1)^{d}=0 .
$$

Let $\Sigma=\left(k_{1}, k_{2}, \cdots, k_{n}\right) \in \mathbb{Z}_{\geq 0}^{n}$. Denote by $n \mid \Sigma$ if $n \mid k_{i}, \forall 1 \leq i \leq n$. For any $r \in \mathbb{Q}$, let $r \Sigma \triangleq\left(r \cdot k_{1}, r \cdot k_{2}, \cdots, r \cdot k_{n}\right) \in \mathbb{Q}^{n}$. 
Theorem 4.1. Let $f, g$ be two functions defined on $\mathbb{Z}_{\geq 0}^{n} \times \mathbb{Z}_{\geq 0}$. $\alpha$ is a function on $\mathbb{Z}_{+}$such that $\alpha(n) \alpha(m)=\alpha(n m)$. If

$$
\sum_{n \mid \Sigma} \alpha(n) f\left(\frac{\Sigma}{n}, n m\right)=g(\Sigma, m)
$$

holds for any $\Sigma \in \mathbb{Z}_{\geq 0}^{n}, m \in \mathbb{Z}_{\geq 0}$. Then we have

$$
f(\Sigma, m)=\sum_{n \mid \Sigma} \mu(n) \alpha(n) g\left(\frac{\Sigma}{n}, n m\right) .
$$

Proof. If $\alpha \equiv 0$, it's trivial. Otherwise, $\alpha(n) \alpha(m)=\alpha(n m)$ implies $\alpha(1)=1$. Note that given $g(\Sigma, m)$ 's, the solution of $f(\Sigma, m)$ in (9) is unique. Hence it's sufficient to show (10) is a solution of (9).

$$
\begin{aligned}
\sum_{n \mid \Sigma} \alpha(n) f\left(\frac{\Sigma}{n}, n m\right) & =\sum_{n \mid \Sigma} \alpha(n) \sum_{k \mid \Sigma} \mu(k) a(k) g\left(\frac{\Sigma}{k n}, k n m\right) \\
& =\sum_{k n \mid d} \alpha(n) \alpha(k) \mu(k) g\left(\frac{\Sigma}{k n}, k n m\right) \\
& =g(\Sigma, m)+\sum_{p>1} \sum_{k n=p} \alpha(n k) \mu(k) g\left(\frac{\Sigma}{k n}, k n m\right) \\
& =g(\Sigma, m)+\sum_{p>1} \alpha(p) g\left(\frac{\Sigma}{p}, p m\right) \sum_{k \mid p} \mu(k) \\
& =g(\Sigma, m)
\end{aligned}
$$

Where in the last step, we've used Lemma 4.1. The proof is completed.

4.2. Explicit formula for local Gopakumar-Vafa invariants. Given a nonsingular, projective, toric, Fano Surface $S$, the topological string amplitudes for the local toric Calabi-Yau manifold $K_{S}$ ([19] 2] 9] [5]) is:

$$
\begin{aligned}
Z_{\text {top str }}^{(S)}= & \sum_{R^{1}, R^{2}, \cdots, R^{N}} \mathcal{W}_{R^{N} R^{1}} \mathcal{W}_{R^{1} R^{2}} \mathcal{W}_{R^{2} R^{3}} \cdots \mathcal{W}_{R^{N-1} R^{N}}(-1)^{\sum_{i=1}^{N} \gamma_{i}\left|R^{i}\right|} \\
& \times q^{\frac{1}{2} \sum_{i=1}^{N} k_{R^{i}} \gamma_{i}} \exp \left\{-\sum_{i=1}^{N} t_{i}\left|R^{i}\right|\right\} \\
= & \sum_{\vec{R}=\left(R^{1}, R^{2}, \cdots, R^{N}\right)} \mathcal{W}_{\vec{R}, \gamma} \exp \left(-\sum_{i=1}^{N} t_{i}\left|R^{i}\right|\right)
\end{aligned}
$$

Where $\gamma_{i}$ is the self-intersection numbers of the rational curves associated to the i-th edge, $t_{i}$ 's are linear combinations of Kähler parameters. In our calculation, $\gamma$ is fixed. We may omit $\gamma$ and write $\mathcal{W}_{\vec{R}}$ instead of $\mathcal{W}_{\vec{R}, \gamma}$. 


$$
\begin{aligned}
F_{\text {closed }} & =\sum_{\Sigma \in H_{2}(S, \mathbb{Z}), \Sigma \neq 0} \sum_{n=1}^{\infty} \sum_{g=0}^{\infty} \frac{\widehat{N}_{\Sigma}^{g}[n]^{2 g-2}}{n} e^{-n \omega \cdot \Sigma} \\
& =\sum_{\Sigma \in H_{2}(S, \mathbb{Z}), \Sigma \neq 0} e^{-\omega \cdot \Sigma} \sum_{n \mid \Sigma} \frac{1}{n} \sum_{g=0}^{\infty} \widehat{N}_{\frac{\Sigma}{n}}^{g}[n]^{2 g-2}
\end{aligned}
$$

Where $\widehat{N}_{\Sigma}^{g}$ 's are the local Gopakumar-Vafa invariants.

Fix a basis in $H_{2}(X, \mathbb{Z})$, one can identify $H_{2}(X, \mathbb{Z})=\mathbb{Z}^{m}$. We only consider $\Sigma=\left(k_{1}, k_{2}, \cdots, k_{m}\right) \in \mathbb{Z}_{\geq 0}^{m}$. Denote by $|\Sigma|=\sum_{i=1}^{m} k_{i}, \tilde{P}_{\Sigma}(x)=$ $\sum_{g=0}^{\infty} \widehat{N}_{\Sigma}^{g} x^{g-1}$. Let $\omega=\left(\omega_{1}, \omega_{2}, \cdots, \omega_{m}\right)^{t}$. Here we write $\omega$ as an $m \times$ 1 matrix, $\Sigma$ a $1 \times m$ matrix. The relation between $T \triangleq\left(t_{1}, t_{2}, \cdots, t_{N}\right)^{t}$ and $\omega$ is $T=A \cdot \omega$, where $A$ is an $N \times m$ matrix. Denote by $|\vec{R}|=$ $\left(\left|R_{1}\right|,\left|R_{2}\right|, \cdots,\left|R_{N}\right|\right)$.

Then

$$
\begin{aligned}
F_{\text {closed }} & =\log \left(1+\sum_{\|\vec{R}\| \geq 1} \mathcal{W}_{\vec{R}} e^{-|\vec{R}| \cdot T}\right) \\
& =\log \left(1+\sum_{d=1}^{\infty} \eta_{d}\right) \\
& =\sum_{\lambda} \theta_{\lambda} \eta_{\lambda}
\end{aligned}
$$

Where $\eta_{d}=\sum_{|\Sigma|=d} \sum_{|\vec{R}| \cdot A=\Sigma} \mathcal{W}_{\vec{R}} \exp (-|\vec{R}| \cdot T), \eta_{\lambda}=\prod_{i \geq 1} \eta_{i}$.

Combine the above two formulas,

$$
\sum_{n \mid \Sigma} \frac{1}{n} \tilde{P}_{\frac{\Sigma}{n}}\left([n]^{2}\right)=\sum_{\lambda \vdash|\Sigma|} \theta_{\lambda}\left\langle\eta_{\lambda}(q)\right\rangle_{\Sigma}
$$

Where $\left\langle\eta_{\lambda}\right\rangle_{\Sigma}$ is the coefficient of $e^{-\omega \cdot \Sigma}$ in $\eta_{\lambda}$. Let $P_{\Sigma}(x)=x \tilde{P}_{\Sigma}(x)=$ $\sum_{g \geq 0} \widehat{N}_{\Sigma}^{g} x^{g}$. By Theorem 4.1, from the above formula we can derive

$$
P_{\Sigma}\left([1]^{2}\right)=[1]^{2} \cdot \sum_{n \mid \Sigma} \frac{\mu(n)}{n} \sum_{\lambda \vdash \frac{|\Sigma|}{n}} \theta_{\lambda}\left\langle\eta_{\lambda}\left(q^{n}\right)\right\rangle_{\frac{\Sigma}{n}}
$$

We fix the following notation in this paper

$$
\mathcal{L}[x]=\left\{\frac{a(x)}{b(x)}: a(x), b(x) \in \mathbb{Z}[x], b(x) \neq 0 \text { and } b(x) \text { is monic }\right\}
$$

Proposition 4.1. $\mathcal{L}[x]$ is a ring with conventional addition and multiplication.

Proof. Trivial calculation. 
By Corollary 3.1 Lemma 3.2 and Proposition 4.1, we have:

Lemma 4.2. $d \in \mathbb{N},\left\langle\eta_{d}(q)\right\rangle_{\Sigma} \in \mathcal{L}[x]$.

Proposition 4.2. $\lambda$ is a partition, let $l=l(\lambda)$, then

$$
\left\langle\eta_{\lambda}(q)\right\rangle_{\Sigma}=\sum_{\Sigma_{1}+\Sigma_{2}+\cdots+\Sigma_{l}=\Sigma}\left\langle\eta_{\lambda_{1}}(q)\right\rangle_{\Sigma_{1}} \cdots\left\langle\eta_{\lambda_{l}}(q)\right\rangle_{\Sigma_{l}}
$$

Then by the above lemma, $\left\langle\eta_{\lambda}(q)\right\rangle_{\Sigma} \in \mathcal{L}[x]$

Proof. By direction calculation.

5. The integrality of local Gopakumar-Vafa invariants

5.1. Pattern theorem. $r \in \mathbb{Q}, p$ is a prime number. Let $r=p^{k} \frac{m}{n}$, where $m, n, k \in \mathbb{Z}, p \nmid m n$. Define $\xi_{p}(r)=k$. Define

$$
\mathcal{R}[x]=\left\{\frac{c(x)}{d(x)}: c(x), d(x) \in \mathbb{Z}[x]\right\}
$$

Definition 5.1. $\forall a(x), b(x) \in \mathcal{R}[x]$. Let

$$
a(x)-b(x)=r \frac{f(x)}{g(x)}
$$

where $r \in \mathbb{Q}, f$ and $g$ are primitive polynomials in $\mathbb{Z}[x]$. Let $p$ be a prime number, $k \in \mathbb{Z}$. Denote by

$$
a(x) \equiv b(x) \bmod \left(p^{k}\right)
$$

if $\xi_{p}(r) \geq k$; denote by

$$
a(x) \Xi b(x) \bmod \left(\frac{1}{p}\right)
$$

if $\xi_{p}(r) \geq 0$.

Remark 5.1. If $a(x) \Xi b(x) \bmod \left(\frac{1}{p}\right), b(x) \Xi c(x) \bmod \left(\frac{1}{p}\right)$. It's easy to see that $a(x) \Xi c(x) \bmod \left(\frac{1}{p}\right)$.

Lemma 5.1. $a, b \in \mathbb{Z}, r \in \mathbb{N}, p$ is a prime number, $f(x) \in \mathbb{Z}[x]$. The following statements hold:
(A) $\frac{a}{\operatorname{gcd}(a, b)} \mid\left(\begin{array}{l}a \\ b\end{array}\right)$.
(B) $p^{r} \mid\left(a^{p^{r}}-a^{p^{r-1}}\right)$.
(C) $f(x) \in \mathbb{Z}[x],(f(x))^{p^{r}} \equiv\left(f\left(x^{p}\right)\right)^{p^{r-1}} \bmod \left(p^{r}\right)$
(D) $g(x) \in \mathcal{L}[x], g(x)^{p^{r}}-g\left(x^{p}\right)^{p^{r-1}}=p^{r} \cdot h(x)$, where $h(x) \in \mathcal{L}[x]$

Proof. (A). $\left(\begin{array}{l}a \\ b\end{array}\right)=\frac{a}{b}\left(\begin{array}{l}a-1 \\ b-1\end{array}\right) \Rightarrow \frac{a}{\operatorname{gcd}(a, b)} \mid\left(\begin{array}{l}a \\ b\end{array}\right)$. 
(B). If $a$ is $p$, the claim is obviously true since $p^{r-1}>r$. If $\operatorname{gcd}(a, p)=1$, by Fermat theorem, $a^{p-1} \equiv 1 \bmod (p)$. Then

$$
\begin{aligned}
a^{p^{r}}-a^{p^{r-1}} & =a^{p^{r-1}}\left((k p+1)^{p^{r-1}(p-1)}-1\right) \\
& =a^{p^{r-1}}\left(\sum_{i=1}^{p^{r-1}}(k p)^{i}\left(\begin{array}{c}
p^{r-1} \\
i
\end{array}\right)\right) \\
& \equiv 0 \bmod \left(p^{r}\right)
\end{aligned}
$$

In the last step, we used (A) and the fact $i>\log (i)$.

(C). By (A) and (B), $(f(x))^{p^{r}}-\left(f\left(x^{p}\right)\right)^{p^{r-1}} \equiv(f(0))^{p^{r}}-(f(0))^{p^{r-1}}$ $\bmod \left(p^{r}\right) \equiv 0 \bmod \left(p^{r}\right)$.

(D). $g(x)=\frac{a(x)}{b(x)}$, where $b(x)$ is monic.

$$
g(x)^{p^{r}}-g\left(x^{p}\right)^{p^{r-1}}=\frac{a(x)^{p^{r}} b\left(x^{p}\right)^{p^{r-1}}-a\left(x^{p}\right)^{p^{r-1}} b(x)^{p^{r}}}{b(x)^{p^{r}} b\left(x^{p}\right)^{p^{r-1}}}
$$

By $(\mathrm{C})$ :

$$
\begin{aligned}
& a(x)^{p^{r}} b\left(x^{p}\right)^{p^{r-1}}-a\left(x^{p}\right)^{p^{r-1}} b(x)^{p^{r}} \\
& \equiv a\left(x^{p}\right)^{p^{r-1}} b\left(x^{p}\right)^{p^{r-1}}-a\left(x^{p}\right)^{p^{r-1}} b(x)^{p^{r}} \bmod \left(p^{r}\right) \\
& \equiv a\left(x^{p}\right)^{p^{r}} b(x)^{p^{r}}-a\left(x^{p}\right)^{p^{r-1}} b(x)^{p^{r}} \bmod \left(p^{r}\right) \\
& \equiv 0 \bmod \left(p^{r}\right)
\end{aligned}
$$

(D) is then proved.

With these preparation, we have the following pattern theorem:

Theorem 5.1. $f_{\Sigma}(x), g_{\Sigma}^{(d)}(x), \psi_{n}(x)$ satisfy the following conditions:

(1) $f_{\Sigma}(x) \in \mathbb{Q}[x]$.

(2) $g_{\Sigma}^{(d)}(x) \in \mathcal{L}[x], \forall d$. Let $\lambda=\left(\lambda_{1}, \cdots, \lambda_{l(\lambda)}\right)$ be a partition, define

$$
g_{\Sigma}^{(\lambda)}=\sum_{\Sigma_{1}+\Sigma_{2}+\cdots+\Sigma_{l(\lambda)}=\Sigma} g_{\Sigma_{1}}^{\left(\lambda_{1}\right)}(x) \cdots g_{\Sigma_{l(\lambda)}}^{\left(\lambda_{l(\lambda)}\right)}(x)
$$

(3) $\psi_{k}(x)$ 's are monic polynomials of degree $k$ such that $\psi_{n p}(x)-\psi_{n}(x)^{p} \equiv$ $0 \bmod (p)$, where $p$ is prime.

(4) The following equation holds:

$$
f_{\Sigma}(x)=x \cdot \sum_{n \mid \Sigma} \frac{\mu(n)}{n} \sum_{\lambda \vdash \frac{|\Sigma|}{n}} \theta_{\lambda} g_{\frac{\Sigma}{n}}^{(\lambda)}\left(\psi_{n}(x)\right)
$$

Then, $f_{\Sigma}(x) \in \mathbb{Z}[x]$.

Proof. Denote by $H_{\Sigma}(x)=\sum_{\lambda \vdash|\Sigma|} \theta_{\lambda} g_{\Sigma}^{(\lambda)}(x)$. Before proceeding the proof, let's look at the following lemma. 
Lemma 5.2. Notations are as above, then for any prime number $p$, we have

$$
H_{p \Sigma}(x)-\frac{1}{p} H_{\Sigma}\left(\psi_{p}(x)\right) \Xi 0 \bmod \left(\frac{1}{p}\right) .
$$

Proof. By (A) in Lemma 5.1 $\theta_{\lambda}$ is not an integer if and only if there exists $k>1$ such that $k \mid m_{i}(\lambda), \forall i$. However, by condition (2), the terms in $H_{\Sigma}$ are not in $\mathcal{L}[x]$ will be when $\lambda=\left(\lambda_{1}^{n_{1}} \cdots \lambda_{k}^{n_{k}}\right)$ and the corresponding term has to be $\pm \frac{m}{l}\left(g_{\Sigma_{1}}^{\left(\lambda_{1}\right)}\right)^{n_{1}} \cdots\left(g_{\Sigma_{k}}^{\left(\lambda_{k}\right)}\right)^{n_{k}}$, where $m$ is some integer, $l=\operatorname{gcd}\left(n_{1}, \cdots, n_{k}\right)$. Otherwise $l$ will be cancelled by the same term with permutations in $H_{\Sigma}$. Obviously, for this term, $n_{1} \Sigma_{1}+\cdots+n_{k} \Sigma_{k}=\Sigma$. Then $l \mid \Sigma$. So $H_{\Sigma}=$ $h(x)+\frac{a(x)}{c \cdot b(x)}$, where $h(x), \frac{a(x)}{b(x)} \in \mathcal{L}[x], c \mid \Sigma$.

First, let's consider $p>2$. Then $p$ is odd. We only consider $1 / p$ factor in the terms of $H_{p \Sigma}$. The number of terms in $H_{p \Sigma}$ which contains factor $1 / p$ is equal to that of $H_{\Sigma}$. We pair them in a way that the general term will be:

$$
\begin{aligned}
& A \triangleq(-1)^{p^{r-1} \cdot a \cdot s} \frac{1}{p^{r} \cdot a}\left\{\zeta \cdot\left(\left(g_{\Sigma_{1}}^{\left(\lambda_{1}\right)}(x)\right)^{s_{1}} \cdots\left(g_{\Sigma_{k}}^{\left(\lambda_{k}\right)}(x)\right)^{s_{k}}\right)\right)^{p^{r} \cdot a} \\
&\left.-\eta \cdot\left(\left(g_{\Sigma_{1}}^{\left(\lambda_{1}\right)}\left(\psi_{p}(x)\right)\right)^{s_{1}} \cdots\left(g_{\Sigma_{k}}^{\left(\lambda_{k}\right)}\left(\psi_{p}(x)\right)\right)^{s_{k}}\right)^{p^{r-1} \cdot a}\right\} \\
& \Xi \quad(-1)^{p^{r-1} \cdot a \cdot s} \frac{\eta}{p^{r} \cdot a}\left(\phi(x)^{p^{r}}-\phi\left(x^{p}\right)^{p^{r-1}}\right) \bmod \left(\frac{1}{p}\right)
\end{aligned}
$$

where $a, b \in \mathbb{Z}$ and $\operatorname{gcd}(p, a)=1$ and

$$
\begin{aligned}
\zeta & =\left(\begin{array}{c}
p^{r} a s \\
p^{r} a s_{1}, p^{r} a s_{2}, \cdots, p^{r} a s_{k}
\end{array}\right) \\
\eta & =\left(\begin{array}{c}
p^{r-1} a s \\
p^{r-1} a s_{1}, p^{r-1} a s_{2}, \cdots, p^{r-1} a s_{k}
\end{array}\right) \\
\phi(x) & =\left(g_{\Sigma_{1}}^{\left(\lambda_{1}\right)}(x)\right)^{a s_{1}} \cdots\left(g_{\Sigma_{k}}^{\left(\lambda_{k}\right)}(x)\right)^{a s_{k}}
\end{aligned}
$$

In (14), we have used Theorem A.1, $\psi_{p}(x) \equiv x^{p} \bmod (p)$ and $p^{r} \mid p^{p^{r-1}}$. By (D) in lemma 5.1, we have $A \Xi 0 \bmod \left(\frac{1}{p}\right)$.

If $p=2$ and $2 \mid \Sigma$, similar to (13), we still get the subtraction. Then we can still use (4) in lemma 5.1 to prove $A \Xi 0 \bmod \left(\frac{1}{2}\right)$.

If $p=2$ and $2 \nmid \Sigma$. Different from (13), it will be the summation of the two terms. But we only have to show $A \Xi 0 \bmod \left(\frac{1}{2}\right)$ with at most $\frac{1}{2}$ appearing, which will be obtained by $2 \mid(1+1)$.

The lemma is proved.

Now, we come back to the proof of the theorem. By the condition (4),

$$
f_{\Sigma}(x)=x \cdot \sum_{n \mid \Sigma} \frac{\mu(n)}{n} \sum_{\lambda \vdash \frac{|\Sigma|}{n}} \theta_{\lambda} g_{\frac{\Sigma}{n}}^{(\lambda)}\left(\psi_{n}(x)\right)
$$

We have $f_{\Sigma}(x)=\frac{a(x)}{c \cdot b(x)}$, where $a(x), b(x) \in \mathbb{Z}[x], c \mid \Sigma$ and $b(x)$ is monic. To see $c \mid \Sigma$, we only notice that in the above formula, $n \mid \Sigma$ and in the term 
$\sum_{\lambda \vdash \frac{|\Sigma|}{n}} \theta_{\lambda} g_{\frac{\Sigma}{n}}^{(\lambda)}\left(\psi_{n}(x)\right)$, if there is any term which is of form $\frac{1}{d} h(x)$, where $h(x) \in \mathcal{L}[x]$, follow the first paragraph of the proof of Lemma [5.2 $d \mid \frac{\Sigma}{n}$.

We will show $f_{\Sigma}(x) \in \mathcal{L}[x]$ by proving: $\forall p \mid \Sigma$ prime, $f_{\Sigma}(x) \Xi 0 \bmod \left(\frac{1}{p}\right)$.

$$
\begin{aligned}
f_{\Sigma}(x) & =x \cdot \sum_{n \mid \Sigma} \frac{\mu(n)}{n} \sum_{\lambda \vdash \frac{|\Sigma|}{n}} \theta_{\lambda} g_{\frac{\Sigma}{n}}^{(\lambda)}\left(\psi_{n}(x)\right) \\
& =x \sum_{n \mid \Sigma, p \nmid n} \frac{\mu(n)}{n} H_{\frac{\Sigma}{n}}\left(\psi_{n}(x)\right)+x \sum_{n \mid \Sigma, p \nmid n} \frac{\mu(p n)}{p n} H_{\frac{\Sigma}{p n}}\left(\psi_{p n}(x)\right) \\
& \Xi \quad x \cdot \sum_{n \mid \Sigma, p \nmid n} \frac{\mu(n)}{n}\left(H_{\frac{\Sigma}{n}}\left(\psi_{n}(x)\right)-\frac{1}{p} H_{\frac{\Sigma}{p n}}\left(\left(\psi_{n}(x)\right)^{p}\right)\right) \bmod \left(\frac{1}{p}\right) \\
& \Xi \quad 0 \bmod \left(\frac{1}{p}\right)
\end{aligned}
$$

Where in the third step, we used the condition $(3)$ and $\mu(p n)=-\mu(n)$; in the last step, lemma 5.2 is applied.

Then $f_{\Sigma}(x)=\frac{a(x)}{b(x)} \in \mathcal{L}[x] . f_{\Sigma}(x) \in \mathbb{Q}[x]$, so we can write $f_{\Sigma}(x)=\frac{1}{n} h(x)$, where $n \in \mathbb{N}, h(x) \in \mathbb{Z}[x]$. Assume $n \nmid h(x)$, then $n>1$. On the one hand,

$$
a(x)=\frac{1}{n} b(x) h(x) .
$$

Since $n \nmid h(x)$, we have $n \mid b(x)$. On the other hand, $b(x)$ is monic, so $b(x)$ is primitive, which is a contradiction. Then $n \mid h(x)$ and $f_{\Sigma}(x) \in \mathbb{Z}[x]$. The proof is completed.

\subsection{Main results.}

Theorem 5.2. $P_{\Sigma}(x)=\sum_{g=0}^{\infty} \widehat{N}_{\Sigma}^{g} x^{g} \in \mathbb{Z}[x]$.

Proof. By (11)

$$
P_{\Sigma}\left([1]^{2}\right)=[1]^{2} \sum_{n \mid \Sigma} \frac{\mu(n)}{n} \sum_{\lambda \vdash \frac{|\Sigma|}{n}} \theta_{\lambda}\left\langle\eta_{\lambda}\left(q^{n}\right)\right\rangle_{\frac{\Sigma}{n}}
$$

Let $x=[1]^{2}=\left(q^{\frac{1}{2}}-q^{-\frac{1}{2}}\right)^{2}, \psi_{n}(x)=[n]^{2}$. First, we show $P_{\Sigma}(x)$ satisfies $(1)$ of the Theorem 5.1. Note that $P_{\Sigma}(x)=\frac{a(x)}{b(x)}$, where $a(x), b(x) \in \mathbb{Z}[x]$. In fact, $b(x)=q^{k} \prod_{i_{s}}\left(1-q^{i_{s}}\right)^{2}$ is a finite product for some $k$ and $i_{s}$ 's, which can be obtained from (6) and (7). As a Laurent series in $q$ for $0<|q|<1, q=0$ is a pole of $\frac{a(x)}{b(x)}$. If there are infinitely many $g$ 's such that $\widehat{N}_{\Sigma}^{g}$ is nonzero for the fixed $\Sigma$, then $q=0$ is an essential singular point of $P_{\Sigma}(x)$, which is a contradiction. So $P_{\Sigma}(x)$ is a polynomial. $\widehat{N}_{\Sigma}^{g}$ 's are rational numbers, which can be easily obtained from the definition. $P_{\Sigma}(x) \in \mathbb{Q}[x]$. 
Then by Proposition 4.2 one finds that our situation is exactly like the Theorem 5.1 as long as we show the functions $\psi_{n}$ 's have the same properties as (3) in the Theorem 5.1 .

By the definition of $\psi_{n}$, we only need to show $\psi_{p}(x)-x^{p} \equiv 0 \bmod (p)$.

$$
\begin{aligned}
{[1]^{2 p} } & =\sum_{k=1}^{p-1}\left(\begin{array}{c}
2 p \\
k
\end{array}\right)(-1)^{k}\left(q^{p-k}+q^{-(p-k)}\right)+\left(q^{p}+q^{-p}-2\right)+\left(\begin{array}{c}
2 p \\
p
\end{array}\right)+2 \\
& =\sum_{k=1}^{p-1}\left(\begin{array}{c}
2 p \\
k
\end{array}\right)(-1)^{k}\left(q^{n-k}+q^{-(n-k)}\right)+[p]^{2}+\left(\begin{array}{c}
2 p \\
p
\end{array}\right)+2
\end{aligned}
$$

equivalently,

$$
[p]^{2}=-\sum_{k=1}^{p-1}\left(\begin{array}{c}
2 p \\
k
\end{array}\right)(-1)^{k}\left(q^{n-k}+q^{-(n-k)}\right)+[1]^{2 p}-\left(\begin{array}{c}
2 p \\
p
\end{array}\right)-2
$$

By lemma 3.3, $q^{n}+q^{-n} \in \mathbb{Z}[x]$, so $[p]^{2} \in \mathbb{Z}[x]$. By (A) of the Lemma 5.1 $p \mid\left(\begin{array}{c}2 p \\ k\end{array}\right)$ when $1 \leq k \leq p-1 .[1]^{2 p} \rightarrow 0$ as $[1]^{2} \rightarrow 0$ implies as a polynomial of $x,[p]^{2}$ has 0 as the constant term. Then $\psi_{p}(x)-x^{p}=[p]^{2}-[1]^{2 p} \equiv 0$ $\bmod (p)$.

The proof is completed.

Remark 5.2. Actually, if one wants, one can precisely calculate the degree of $P_{\Sigma}(x)$ using formula (11) and the following formula about Schur function.

$$
S_{\lambda}(x)=\sum_{|\rho|=|\lambda|} \frac{\chi_{\lambda}(\rho)}{z_{\rho}} p_{\rho}(x) .
$$

where $\chi_{\rho}$ is the character of representation $\rho$.

\section{Some examples}

We may leave the results of Hirzebruch surfaces $\mathbb{F}_{k}$ and $\mathbb{P}^{2}$ as the following theorem. We use the same notation as those in the introduction: $P_{\Sigma}^{(S)}(x)=\sum_{g \geq 0} \widehat{N}_{\Sigma}^{g}(S) x^{g}$, where $\widehat{N}_{\Sigma}^{g}(S)$ is the local Gopakumar-Vafa invariant of the given class $\Sigma \in H_{2}(S, \mathbb{Z})$ for the Fano surface $S$. Let $A_{\Sigma}(S)$ be the arithmetic genus of the curve with the degree $\Sigma \in H_{2}(S, \mathbb{Z}) . L(\Sigma)$ denotes the holomorphic line bundle associated to the devisor $\Sigma$.

Theorem 6.1. We have

(1) $\operatorname{deg}\left(P_{d}^{\left(\mathbb{P}^{2}\right)}(x)\right)=A_{d}\left(\mathbb{P}^{2}\right)=\frac{1}{2}(d-1)(d-2)$.

$$
\operatorname{deg}\left(P_{(d, m)}^{\left(\mathbb{F}_{k}\right)}(x)\right)=A_{(d, m)}\left(\mathbb{F}_{k}\right)=(d-1)\left(m-1-\frac{k d}{2}\right),
$$


(2) Moreover,

$$
\begin{aligned}
\widehat{N}_{d}^{A_{d}\left(\mathbb{P}^{2}\right)}\left(\mathbb{P}^{2}\right) & =(-1)^{d} \chi(L(\Sigma)) \\
& =\frac{(-1)^{d}}{2}(d+1)(d+2), \\
\widehat{N}_{(d, m)}^{A_{(d, m)}\left(\mathbb{F}_{k}\right)}\left(\mathbb{F}_{k}\right) & =(-1)^{d k} \chi(L(\Sigma)) \\
& =(-1)^{d k}(d+1)\left(m+1-\frac{k d}{2}\right) .
\end{aligned}
$$

Proof. We do computation for $\mathbb{P}^{2}$ as an example. Let $H \in H_{2}\left(\mathbb{P}^{2}, \mathbb{Z}\right)$ be the generator, it satisfies $H \cdot H=1$. So the topological string amplitudes will be

$$
\begin{aligned}
Z_{\text {top str }}^{\left(\mathbb{P}^{2}\right)}= & \sum_{R^{1}, R^{2}, R^{3}} \mathcal{W}_{R^{1} R^{2}} \mathcal{W}_{R^{2} R^{3}} \mathcal{W}_{R^{3} R^{1}}(-1)^{\left|R^{1}\right|+\left|R^{2}\right|+\left|R^{3}\right|} q^{\frac{1}{2} \sum_{i=1}^{3} k_{R^{i}}} \\
& \times \exp \left\{t_{H}\left(\left|R^{1}\right|+\left|R^{2}\right|+\left|R^{3}\right|\right)\right\}
\end{aligned}
$$

We need to study the following term

$$
\mathcal{W}_{R^{1} R^{2}} \mathcal{W}_{R^{2} R^{3}} \mathcal{W}_{R^{3} R^{1}}(-1)^{d} q^{\frac{1}{2} \sum_{i=1}^{3} k_{R^{i}}}
$$

under the condition $\left|R^{1}\right|+\left|R^{2}\right|+\left|R^{3}\right|=d$. Now

$$
\mathcal{W}_{\lambda \mu}=q^{-\frac{|\lambda|+|\mu|}{2}} S_{\lambda}\left(q^{\alpha}\right) S_{\mu}\left(q^{\lambda+\alpha}\right)
$$

By (16] page 44 ex.1)

$$
S_{\lambda}\left(q^{\alpha}\right)=q^{-n(\lambda)} \prod_{x \in \lambda} \frac{1}{1-q^{-h(x)}}
$$

We also have

$$
S_{\mu}\left(q^{\lambda+\alpha}\right)=\sum_{|\rho|=|\mu|} \frac{\chi_{\mu}(\rho)}{z_{\rho}} p_{\rho}
$$

Here we can see degree of $q$ in $S_{\mu}\left(q^{\lambda+\alpha}\right)$ is not greater than $|\lambda| \cdot|\mu|$ by writing down $p_{\rho}$ explicitly and equality holds when $\lambda=(k)$ for $k=|\lambda|$. Hence the degree of $q$ in (15) is no more than

$$
\left|R^{1}\right|\left|R^{2}\right|+\left|R^{2}\right|\left|R^{3}\right|+\left|R^{3}\right|\left|R^{1}\right|-d+\frac{1}{2} \sum_{i=1}^{3} k_{R^{3}}
$$


while

$$
\begin{aligned}
\frac{1}{2} \sum_{i=1}^{3} k_{R^{i}} & =\sum_{i=1}^{3}\left(n\left(\left(R^{i}\right)^{t}\right)-n\left(R^{i}\right)\right) \\
& \leq \sum_{i=1}^{3} n\left(\left(R^{i}\right)^{t}\right) \\
& \leq \sum_{i=1}^{3} \frac{\left|R^{i}\right| \cdot\left(\left|R^{i}\right|-1\right)}{2}
\end{aligned}
$$

The last inequality is obtained from $n\left(\lambda^{t}\right) \leq n((m))$ where $m=|\lambda|$. This can be proved in the following way. Let $l=l(\lambda)$. If one subtracts one from $\lambda_{l}$ and adds one to $\lambda_{1}$, one will find $n\left(\lambda^{t}\right)$ is increasing. So we have the inequality. The equality holds when $\lambda=(m)$.

Let $\left|R^{i}\right|=x_{i}$. Then $\sum x_{i}=d$. In the above inequalities, all equality hold when the corresponding partition is of length 1 . So the $\operatorname{deg} P_{d}-1$ is

$$
\begin{aligned}
& \sum_{i=1}^{3} \frac{x_{i} \cdot\left(x_{i}-1\right)}{2}-d+\left(x_{1} x_{2}+x_{2} x_{3}+x_{3} x_{1}\right) \\
= & \frac{d^{2}-3 d}{2}
\end{aligned}
$$

The coefficient of the highest degree in $P_{\Sigma}$ is the number of the non-negative integer solutions of $x_{1}+x_{2}+x_{3}=d$ multiplied by $(-1)^{d}$, which is $\frac{(-1)^{d}}{2}(d+$ 1) $(d+2)$.

We do the similar calculation and get the degree of $P_{(d, m)}^{\left(\mathbb{F}_{k}\right)}$. The coefficient of the highest order term is the number of non-negative integer solutions of $x_{1}+x_{3}+k x_{4}=m, x_{2}+x_{4}=d$ multiplied by $(-1)^{d k}$, which is $(-1)^{d k} \frac{(d+1)(m+1-k d)}{2}$.

Now we can easily verify the theorem by adjunction formula and HirzebruchRiemann-Roch theorem.

\section{Appendix A.}

Theorem A.1. $\sum_{i=1}^{n} a_{i}=a, p$ is prime, $r \geq 1$. Then

$$
\left(\begin{array}{c}
p^{r} a \\
p^{r} a_{1}, \cdots, p^{r} a_{n}
\end{array}\right)-\left(\begin{array}{c}
p^{r-1} a \\
p^{r-1} a_{1}, \cdots, p^{r-1} a_{n}
\end{array}\right) \equiv 0 \bmod \left(p^{2 r}\right)
$$

Lemma A.1. $p$ is prime, $r \geq 1$. Then

$$
\left(\begin{array}{l}
p^{r} a \\
p^{r} b
\end{array}\right)-\left(\begin{array}{l}
p^{r-1} a \\
p^{r-1} b
\end{array}\right) \equiv 0 \bmod \left(p^{2 r}\right)
$$


Proof.

$$
\begin{aligned}
\frac{\left(\begin{array}{c}
p^{r} a \\
p^{r} b
\end{array}\right)}{\left(\begin{array}{c}
p^{r-1} a \\
p^{r-1} b
\end{array}\right)} & =\frac{\prod_{k=1}^{p^{r} b} \frac{(a-b) p^{r}+k}{k}}{\prod_{k=1}^{p^{r-1} b} \frac{(a-b) p^{r-1}+k}{k}} \\
& =\prod_{\operatorname{gcd}(k, p)=1,1 \leq k \leq p^{r} b} \frac{(a-b) p^{r}+k}{k} \\
& =\prod_{\operatorname{gcd}(k, p)=1,1 \leq k \leq p^{r} b}\left(1+\frac{a-b}{k} p^{r}\right) \\
& \equiv 1+p^{r}(a-b) \sum_{\operatorname{gcd}(k, p)=1,1 \leq k \leq p^{r} b} \frac{1}{k} \bmod \left(p^{2 r}\right)
\end{aligned}
$$

Let $A_{p}(n)=\sum_{1 \leq k \leq n, \operatorname{gcd}(k, p)=1} \frac{1}{k}$. Therefore the proof of the lemma is completed by showing $A_{p}\left(p^{r} b\right)=\frac{p^{r} c}{d}$ for some $c, d$ such that $\operatorname{gcd}(d, p)=1$.

If $\operatorname{gcd}(k, p)=1$, there exist $\alpha_{k}, \beta_{k}$ such that

$$
\alpha_{k} k+\beta_{k} p^{r}=1
$$

Let $B_{p}(n)=\sum_{1 \leq k \leq n, \operatorname{gcd}(k, p)=1} k$. Then by the above formula

$$
\begin{aligned}
A_{p}\left(p^{r} b\right) & \equiv b A_{p}\left(p^{r}\right) \bmod \left(p^{r}\right) \\
& \equiv b B_{p}\left(p^{r}\right) \bmod \left(p^{r}\right)
\end{aligned}
$$

And

$$
\begin{aligned}
B_{p}\left(p^{r}\right) & =\sum_{k=1}^{p^{r}} k-p \sum_{k=1}^{p^{r-1}} k \\
& =\frac{p^{r}\left(p^{r}+1\right)}{2}-p \frac{p^{r-1}\left(p^{r-1}+1\right)}{2} \\
& =\frac{p^{2 r-1}(p-1)}{2}
\end{aligned}
$$

$2 r-1 \geq r$ since $r \geq 1$. The proof is completed.

Now, let's turn to the proof of Theorem A.1.

Proof.

$$
\begin{aligned}
& \left(\begin{array}{c}
p^{r} a \\
p^{r} a_{1}, \cdots, p^{r} a_{n}
\end{array}\right)-\left(\begin{array}{c}
p^{r-1} a \\
p^{r-1} a_{1}, \cdots, p^{r-1} a_{n}
\end{array}\right) \\
= & \prod_{k=1}^{n}\left(\begin{array}{c}
p^{r}\left(a-\sum_{i=1}^{k-1} a_{i}\right) \\
p^{r} a_{k}
\end{array}\right)-\prod_{k=1}^{n}\left(\begin{array}{c}
p^{r-1}\left(a-\sum_{i=1}^{k-1} a_{i}\right) \\
p^{r-1} a_{k}
\end{array}\right) \\
\equiv & 0 \bmod \left(p^{r}\right)
\end{aligned}
$$

In the last step, we have used Lemma A.1. The proof is then completed. 


\section{REFERENCES}

[1] M. Aganagic, A. Klemm, M. Mariño and C. Vafa, "The topological vertex", hep-th/0305132

[2] M. Aganagic, M. Mariño, Vafa, "All loop topological string amplitudes from Chern-Simons theory", Commun.Math.Phys. 247:467-512, 2004. e-Print Archive: hep-th/0206164

[3] T.-M. Chiang, A. Klemm, S.-T. Yau, E. Zaslow, "Local Mirror Symmetry: Calculations and Interpretations", hep-th/9903053

[4] Duiliu-Emanuel Diaconescu, Bogdan Florea, Antonella Grassi, "Geometric transitions, DEL PEZZO surfaces and open string instantons", Adv.Theor.Math.Phys.6:643-702,2003 e-Print Archive: hep-th/0206163

[5] Tohru Eguchi, Hiroaki Kanno, "Topological Strings and Nekrasov's formulas", hep-th/0310235

[6] Rajesh Gopakumar, Cumrun Vafa, "M-theory and topological strings-I", hep-th/9809187

[7] Rajesh Gopakumar, Cumrun Vafa, "M-theory and topological strings-II", hep-th/9812127

[8] Rajesh Gopakumar, Cumrun Vafa, "On the gauge theory/goemetry correspondence", hep-th/9811131 Adv. Theor. Math. Phys. 3 (19999) 1415

[9] Amer Iqbal, "All Genus Topological String Amplitudes and 5-brane Webs as Feynman Diagrams", hep-th/0207114

[10] Amer Iqbal, Amir-Kian Kashani-Poor, "Instanton Counting and Chern-Simons Theory", hep-th/0212279

[11] S. Katz, A. Klemm, C.Vafa, "M-theory, topological strings and spinning black holes", hep-th/9910181 Adv. Theor. Math. Phys. 3 (1999) 1445

[12] J. Li, C.-C. Liu, K. Liu, J. Zhou, "A mathematical theory of the Topological Vertex", math.AG/0408426

[13] J. Li, K. Liu, J. Zhou, "Topological partition function as equivariant indices", private conversation.

[14] C.-C. Liu, K. Liu, J. Zhou, "A proof of a conjecture of Mariño-Vafa on Hodge integrals", J. Differential Geom. 65(2003), NO. 2, 289-340

[15] C.-C. Liu, K. Liu, J. Zhou, "A formula of two-partition Hodge integrals", math.AG/0310272

[16] I. G. MacDonald, "Symmetric Functions and Hall Polynomials", 2nd edition, Clarendon Press, 1995

[17] Nikita A. Nekrasov, "Seiberg-Witten Prepotential from Instanton Counting", hep-th/0306211

[18] Jian Zhou, "Localizations on moduli spaces and free field realizations of Feynman rules", math.AG/0310283

[19] Jian Zhou, "Curve counting and instanton counting", math.AG/0311237

Department of Mathematics, UCLA

E-mail address: ppeng@math.ucla.edu 